\title{
Preface to the special issue on mechanics of materials
}

\author{
Ratna Kumar Annabattula ${ }^{1} \cdot$ Eswara Prasad Korimilli $^{2} \cdot$ Naresh Varma Datla $^{3}$
}

Published online: 26 March 2021

(C) Indian Institute of Technology Madras 2021

Materials play a vital role in the technological advancement of human society. The successful design and performance of various engineering components/structures rely on the fundamental understanding of the underlying material behaviour's mechanics and mechanisms. Hence, studying the mechanics of materials has been an exciting field of research ever since human civilisation started using materials. The study of mechanics of materials continues to be an important field of research due to the challenges offered by the dynamic societal needs. In this context, we take great pleasure in bringing out this special issue on the mechanics of materials. The special issue includes ten articles focusing on contemporary issues relating to various aspects of mechanics of materials, including analytical, numerical and experimental works. A brief overview of the published manuscripts is presented below in the same order as they appear in the special issue.

Ratna Kumar Annabattula

ratna@iitm.ac.in

Eswara Prasad Korimilli

eswar@iiti.ac.in

Naresh Varma Datla

datla@mech.iitd.ac.in

1 Department of Mechanical Engineering, Indian Institute of Technology Madras, Sardar Patel Road, Chennai 600036, India

2 Department of Metallurgy Engineering and Materials Science, Indian Institute of Technology Indore, Khandwa Road, Simrol, Indore, Madhya Pradesh 453552, India

3 Department of Mechanical Engineering, Indian Institute of Technology Delhi, Hauz Khas, New Delhi 110016, India
1. Rate-independent elasto-plastic materials-a brief history and some new developments: In this invited article, the authors present a brief history of developing small-deformation elasto-plasticity models and their evolution into models suitable for applications from fatigue to metal forming. The authors present a promising new approach that requires only the essential features of plasticity, such as rate independence and hysteresis.

2. On the viscoelastic dynamic beam modelling: Motivated by the recent interest in developing bioinspired underwater robotic systems, the authors present a material model capable of describing a robotic fish's dynamic behaviour. The authors use the well-known Kelvin-Voigt model to develop a precise material model of a fish accounting for fluidstructure interactions.

3. Impact of hygrothermal environment on the bistability of variable stiffness laminates with curvilinear fibre paths: In this article, the authors investigate the impact of the hygrothermal environment on the stability of variable stiffness composite shell structures. The authors have developed a semi-analytical model, and their analytical results are compared with finite element simulations performed in commercial finite element software, ABAQUS.

4. Hot deformation characteristics and microstructure evolution of Ti-5Al-3Mo-1.5V alloy: In this experimental work, the authors present the concurrent effect of temperature and strain rate on the microstructure evolution in Ti-5Al-3Mo-1.5V dualphase Titanium alloy. The authors identify that this particular alloy's safe hot working regime is $1248 \mathrm{~K}$ at $10^{-3} \mathrm{~s}^{-1}$.

5. Role of domain configurations on the mechanistic modelling of indentation size effects (ISE) in 
nanohardness of hard and soft PZT piezoceramics: In this experimental work, the authors investigated the role of various domain configurations on the indentation size effects in determining the nanohardness of a famous piezo-ceramic, PZT extensively used for MEMS/NEMS applications.

6. An approach to model mechanical damage in particulate composites with viscoelastic matrix: This article presents a phenomenological model to describe the mechanical damage in a particulate composite with a viscoelastic polymer matrix. They use a dissipation potential based on the effective stored elastic energy density to derive a condition for implicit damage evolution. The authors captured the onset of stress hardening immediately after the damage and subsequent softening response through their work.

7. Morphological characterisation of $2 \mathrm{D}$ packing with bi-disperse particles: In this paper, the authors present a numerical packing method combining particle size growth and dynamic vibration. Their DEM simulation results compare well with equivalent experiments. The morphological structure of the $2 D$ granular assembly is characterised by bond orientational order and pair distribution functions.

8. Near-surface soils: discrete element modeling of selfsupported unconfined drained sand specimens: In this article, the authors present discrete element modelling of dry granular systems subjected to different compaction levels. They have shown experimental results and carefully designed simulations to describe the microscopic mechanisms otherwise not accessible through experiments. The authors study particle shape (spherical/nonspherical) and inter-particle interactions on the force transfer and macroscopic response.

9. Compaction mechanics of a polydisperse crushable spherical granular assembly using discrete element method: In this article, the authors investigate the response of a crushable polydisperse granular system through DEM simulations. In particular, they study the influence of the coefficient of friction, packing fraction and particle size distribution on the macroscopic response of the assembly.

10. Seismic fragility analysis in the probabilistic performance-based earthquake engineering framework: an overview: In this review article, the authors present an overview of the recent developments in the numerical simulation based seismic fragility analysis in probabilistic performance-based earthquake engineering.

We thank the authors for contributing their work to this special issue. A special thanks to all the reviewers for their time and effort, which helped us immensely in making the final decision about the suitability of the articles for publication in this journal and improving the quality of the publications. The Springer publishing team has been very helpful in bringing out this issue despite the challenges amidst COVID-19. We truly appreciate their hard work and professional help. We thank the Editor-in-Chief Prof. Harishankar Ramachandran, and Prof. K. Ramamurthy, Department of Civil Engineering, Indian Institute of Technology Madras, to encourage us to bring out this special issue. We hope that the readers will find the articles published in this special issue exciting and valuable in advancing the knowledge base of mechanics of materials. 